\title{
Логика в онтологии процессов
}

\author{
Владимир Иванович Шалак \\ Институт философии РАН. \\ Российская Федерация, 109240, г. Москва, ул. Гончарная, д. 12, стр. 1. \\ E-mail: shalack@gmail.com
}

\begin{abstract}
Аннотация: Целью работы является исследование языковых средств, которые могут быть использованы для проведения рассуждений о процессуальной онтологии, которая отличается от субстанциальной более сложной структурой элементарных «кирпичиков», лежащих в ее основе. Следствием этого является необходимость использовать языки с более богатыми выразительными возможностями, чем язык классической логики. В работе рассматриваются языки с унарными и бинарными модальностями. Отдельные процессы можно анализировать с двух точек зрения - статики и динамики. В первом случае нас интересуют высказывания, истинность которых зависит лишь от внутренней структуры текущего процесса. Отношение деления процессов позволяет определить на его частях транзитивный, плотный линейный порядок без первого и последнего элементов, который можно понимать как локальный временной порядок данного процесса. Это, в свою очередь, позволяет говорить о начале процессов, их протекании и окончании. Появляется возможность определить понятия состояния процесса и процесса как события. Включение в язык бинарной модальности типа некоммутативной конъюнкции позволяет определить аналог причинно-подобной условной связи между частями процесса. Динамика процессов подразумевает возможность их продолжения за пределы текущего процесса. Это может происходить либо благодаря имплицитным причинным связям, когда текущий процесс содержит в себе причину своего будущего продолжения, либо в результате действий активных агентов, которые сами формируют это продолжение. Для представления динамики процессов как следствия действий активных агентов использован упрощенный вариант языка динамической логики. Также показано, каким образом можно учитывать цели, которые ставят перед собой активные агенты при выполнении тех или иных действий.
\end{abstract}

Ключевые слова: процесс, онтология процессов, логика процессов, статика процессов, динамика процессов

Для цитирования: Шалак В.И. Логика в онтологии процессов // Логические исследования / Logical Investigations. 2021. Т. 27. № 2. C. 48-65. DOI: 10.21146/2074-1472-2021$27-2-48-65$ 


\section{1. Введение}

Целью настоящей работы является исследование языковых средств и логики, которые могут быть использованы для рассуждений о процессуальной онтологии.

В предыдущей публикации Шалак, 2020] был проведен сравнительный логический анализ субстанциальной и процессуальной онтологий. Для процессуальной онтологии были выделены ее основные составляющие структуры и предложен ряд характеризующих их постулатов.

Процессуальную онтологию можно представить четверкой $\langle\boldsymbol{W}, \boldsymbol{U}, \boldsymbol{D}, \leq\rangle$, где $\boldsymbol{W}$ - действительный мир, понимаемый как единый процесс. Предметы в самом общем смысле понимаются как «пучки» составляющих их подпроцессов. В соответствии с этим $\boldsymbol{U}$ представляет совокупность подпроцессов $\boldsymbol{W}$. $\leq$ - отношение частичного порядка на $\boldsymbol{U}$, понимаемое как отношение бъть подпроцессом, а $\boldsymbol{W}$ - его наибольший элемент. Процессы характеризуются протяженностью и направленностью. Протяженность предполагает возможность (мысленного) деления процессов на последовательные части. Это деление представлено трехместным отношением $\boldsymbol{D} \subseteq \boldsymbol{U} \times \boldsymbol{U} \times \boldsymbol{U}: \boldsymbol{D} p q r$ - процесс $p$ можно разделить на предшествующую и последующую части $-q$ и $r$.

Ранее было показано, что отношение $\leq$ естественным образом приводит к логикам силлогистического типа. Теперь мы должны продолжить и исследовать логические структуры, связанные с протяженностью и направленностью процессов.

По сравнению с предыдущей публикацией [Шалак, 2020] семантические постулаты для отношения $\boldsymbol{D}$ уточнены и их стало меньше. Незначительные изменения коснулись постулатов 2, 6 и 7. Для краткости мы используем в метаязыке очевидные по смыслу сокращения $\sim, \&, \vee, \Rightarrow, \Leftrightarrow, \exists, \forall$ для соответствующих фраз естественного языка.

\section{1. Семантические постулаты для отношения $D$}

1. $\forall p \exists x \exists y \boldsymbol{D} p x y$ - всякий процесс $p$ можно разделить на предшествующую $x$ и последующую $y$ части, также являющиеся процессами.

2. $\boldsymbol{D} p x y \Rightarrow \sim \exists q \boldsymbol{D} q y \boldsymbol{x}$ - левую и правую части деления процесса нельзя поменять местами.

3. $\boldsymbol{D} p x y$ \& $\boldsymbol{D} q x y \Rightarrow p=q$ - процесс однозначно задается своими частями.

4. $\boldsymbol{D} p x y$ \& $\boldsymbol{D} p z y \Rightarrow x=z$ - процесс однозначно задается своими частями. 
5. $\boldsymbol{D} p x y \& \boldsymbol{D} p x z \Rightarrow y=z-$ процесс однозначно задается своими частями.

6. $\exists w(\boldsymbol{D} p x w \& \boldsymbol{D} w y z) \Leftrightarrow \exists u(\boldsymbol{D} p u z \& \boldsymbol{D} u x y)$ - последовательное деление процесса на части ассоциативно. Сначала мы можем разделить процесс $p$ на две части $x$ и $w$, а потом разделить вторую часть $w$ еще на две - $y$ и $z$, а можно сначала разделить $p$ на две части $u$ и $z$, а потом разделить первую часть $u$ на $x$ и $y$. Результат останется прежним.

7. $\exists p(\boldsymbol{D} p x y \& \boldsymbol{D} p z u) \Leftrightarrow \exists v(\boldsymbol{D} z x v \& \boldsymbol{D} y v u) \vee \exists v(\boldsymbol{D} x z v \& \boldsymbol{D} u v y) \vee \exists p(x=$ $z \& y=u \& \boldsymbol{D} p x u)$ - как могут соотноситься два произвольных деления одного и того же процесса.

Понятие процесса в процессуальной онтологии является исходным и не редуцируемым ни к чему другому, но это не означает, что мы не можем построить для постулатов стандартную модель, единственное назначение которой - подтвердить их непротиворечивость.

- Пусть $\boldsymbol{W}$ - множество всех рациональных чисел.

- Для любых двух таких рациональных чисел $a$ и $b$, что $a<b$, существует открытый интервал $(a, b)=\{c: a<c<b\}$. Пусть $\boldsymbol{U}=\{(a, b): a \in \boldsymbol{W} \& b \in \boldsymbol{W} \& a<b\} \cup\{\boldsymbol{W}\}$.

- Любое число $c \in(a, b)$ задает деление этого интервала на два других открытых интервала $(a, c)$ и $(c, b)$. Это и будет отношением деления процессов $\boldsymbol{D}=\{<x, y, z>: \exists a \exists b \exists c[a<c<b \& x=(a, b) \& y=$ $(a, c) \& z=(c, b)]\}$.

- Отношение быть подпроцессом отождествим с нестрогим отношением включения $\leq=\{\langle x, y\rangle: x \in \boldsymbol{U} \& y \in \boldsymbol{U} \& x \subseteq y\}$.

Легко проверить, что все наши постулаты истинны в этой модели. При этом необходимо еще раз подчеркнуть, что предложенная модель не означает редукции онтологии процессов к теоретико-множественной субстанциальной онтологии, а всего лишь служит цели показать, что постулаты непротиворечивы. Мы знаем, что логику предикатов нельзя редуцировать к логике высказываний, но одним из способов показать ее непротиворечивость является построение перевода всех формул языка логики предикатов в язык логики высказываний путем стирания кванторов, индивидных констант и переменных. В результате все аксиомы логики предикатов превращаются в тавтологии логики высказываний. На семантическом уровне это равносильно тому, что для доказательства непротиворечивости логики 
предикатов достаточно проверить истинность ее аксиом в одноэлементной модели. Так же и в нашем случае. Для описания и рассуждений о процессах мы вынуждены пользоваться тем фрагментом естественного языка, который имеет теоретико-множественную семантику. Этот язык обязан подчиняться требованию непротиворечивости. Мы всего лишь показали, что принятие постулатов для $\boldsymbol{D}$ не нарушает этого требования.

\section{2. Основные характеристики отношения $D$}

В дальнейшем нам понадобятся определения следующих отношений:

Def.1 $\boldsymbol{L} p x \Leftrightarrow_{d e f} \exists y \boldsymbol{D} p x y$, где $x$ является левой частью (началом) процесса $p$.

Def.2 $\boldsymbol{R} p x \Leftrightarrow_{\text {def }} \exists y \boldsymbol{D} p y x$, где $x$ является правой частью (концом) процесса $p$.

$D e f .3 \boldsymbol{S p x} \Leftrightarrow_{d e f} \exists y(\boldsymbol{R} p y \& \boldsymbol{L} y x)$, где $x$ является внутренним сегментом процесса $p$.

Def.4 $\boldsymbol{P} p x \Leftrightarrow_{\text {def }} \boldsymbol{L} p x \vee \boldsymbol{S} p x \vee \boldsymbol{R} p x$, где $x$ является собственной частью процесса $p$.

Основные характеристики логики процессов определяются свойствами отношения $\boldsymbol{D}$ и производных от него, которые мы только что определили.

Утверждение 1 . Отношения $\boldsymbol{D}, \boldsymbol{L}, \boldsymbol{R}$ и $\boldsymbol{P}$ обладают следующими свойствами:

1. $\boldsymbol{D} p x y \Rightarrow p \neq x \& p \neq y \& x \neq y$.

2. $\sim \boldsymbol{L} x \boldsymbol{x}$ - иррефлексивность.

3. $\boldsymbol{L} x y \& \boldsymbol{L} y z \Rightarrow \boldsymbol{L} x z-$ транзитивность.

4. $\boldsymbol{L} x y \Rightarrow \exists z(\boldsymbol{L} x z \& \boldsymbol{L} z y)-$ плотность.

5. $\boldsymbol{L} p x \& \boldsymbol{L} p y \Rightarrow \boldsymbol{L} x y \vee \boldsymbol{L} y x \vee x=y$ - линейность.

6. $\forall x \exists y \boldsymbol{L} x y-$ нет последнего элемента.

7. $\sim \boldsymbol{R} x x-$ иррефлексивность.

8. $\boldsymbol{R} x y \& \boldsymbol{R} y z \Rightarrow \boldsymbol{R} x z-$ транзитивность.

9. $\boldsymbol{R} x y \Rightarrow \exists z(\boldsymbol{R} x z \& \boldsymbol{R} z y)-$ плотность. 
10. $\boldsymbol{R} p x \& \boldsymbol{R} p y \Rightarrow \boldsymbol{R} x y \vee \boldsymbol{R} y x \vee x=y-$ линейность.

11. $\forall x \exists y \boldsymbol{R} x y-$ нет последнего элемента.

12. $\boldsymbol{L} p x \Rightarrow \sim \boldsymbol{R} p x$ - дизъюнктность $\boldsymbol{L}$ и $\boldsymbol{R}$.

13. $\sim \boldsymbol{P} x x-$ иррефлексивность.

14. $\forall x \exists y \boldsymbol{P} x y-$ нет последнего элемента.

15. $\boldsymbol{P} x y \& \boldsymbol{P} y z \Rightarrow \boldsymbol{P} x z-$ транзитивность.

16. $\boldsymbol{P} x y \Rightarrow \exists z(\boldsymbol{P} x z \& \boldsymbol{P} z y)-$ плотность.

Поскольку в настоящей работе нас будут в основном интересовать те характеристики логики процессов, которые связаны с их протяженностью и направленностью, в определении модели $\boldsymbol{M}=\langle\boldsymbol{U}, \boldsymbol{D}, \boldsymbol{I}\rangle$ мы опустим упоминание отношения бытъ подпрочессом и наибольшего элемента $\boldsymbol{W}$ для него, оставив лишь множество всех процессов $\boldsymbol{U}$, отношение деления процессов $\boldsymbol{D}$ и добавив функцию интерпретации $\boldsymbol{I}: \operatorname{Prop} \rightarrow 2^{\boldsymbol{U}}$, сопоставляющую каждой пропозициональной переменной множество процессов, на которых она истинна. Определения обычных связок оставим стандартными.

\section{2. Статика процессов}

Обычно для выражения протяженности процессов мы прибегаем к использованию временных маркеров было или будет. В онтологии процессов время пока что никак не фигурирует, но, как следствие нашего понимания протяженности, условно левые и правые части могут быть ассоциированы с началом и концом процесса относительно данного деления. Это и послужит нашей отправной точкой.

\section{1. Процессы и время}

Расширим язык логики высказываний операторами $\langle L\rangle,\langle R\rangle$ и определим понятие формулы следующим образом:

$$
\text { Def.5 } A:=\operatorname{Prop}|\neg A|(A \wedge B)|\langle L\rangle A|\langle R\rangle A .
$$

Моделью будем называть тройку $\boldsymbol{M}=\langle\boldsymbol{U}, \boldsymbol{D}, \boldsymbol{I}\rangle$.

Def.6 $\boldsymbol{M}, x \vDash A-$ формула $A$ истинна на процессе $x$ модели $\boldsymbol{M}$ :

- $M, x \vDash q \Leftrightarrow x \in I(q), q \in$ Prop;

- $M, x \vDash \neg A \Leftrightarrow M, x \nvdash \models A$; 
- $M, x \vDash A \wedge B \Leftrightarrow M, x \vDash A \& M, x \vDash B$;

- $\boldsymbol{M}, x \vDash\langle L\rangle A \Leftrightarrow \exists y(\boldsymbol{L} x y \& \boldsymbol{M}, y \vDash A$;

- $\boldsymbol{M}, x \vDash\langle R\rangle A \Leftrightarrow \exists y(\boldsymbol{R} x y \& \boldsymbol{M}, y \vDash A)$.

$D e f .7 \vDash A \Leftrightarrow_{\operatorname{def}} \forall \boldsymbol{M} \forall x(\boldsymbol{M}, x \vDash A)-$ формула $A$ общезначима.

В дальнейшем, если модель $\boldsymbol{M}$ фиксирована, мы будем опускать ее упоминание. Смысл формул вида $\langle L\rangle A$ и $\langle R\rangle A$ достаточно очевиден. Формула $\langle L\rangle A$ истинна на процессе $x$, е. и т.е. $A$ истинна на некотором его начале. Аналогично для $\langle R\rangle A$. Условия истинности формул $\langle L\rangle A$ и $\langle R\rangle A$ определяются аналогично условиям истинности формул с операторами возможности в модальной логике, если $\boldsymbol{L} x y$ и $\boldsymbol{R} x y$ понимать как отношения достижимости на возможных мирах. Это позволяет по аналогии с модальной логикой определить дуальные операторы $[L]$ и $[R]$ типа необходимости:

Def.8 $[L] A \Leftrightarrow \operatorname{def} \neg\langle L\rangle \neg A$.

Def.9 $[R] A \Leftrightarrow \Leftrightarrow_{\text {def }} \neg\langle R\rangle \neg A$.

Им будут соответствовать следующие условия истинности:

- $x \vDash[L] A \Leftrightarrow \forall y(\boldsymbol{L} x y \Rightarrow y \vDash A)$;

- $x \vDash[R] A \Leftrightarrow \forall y(\boldsymbol{R} x y \Rightarrow y \vDash A)$.

Имеет место следующее утверждение.

\section{Утверждение 2.}

1. $\vDash A \Rightarrow \vDash[L] A$.

2. $\vDash[L](A \supset B) \supset([L] A \supset[L] B)$.

3. $\vDash[L] A \equiv \neg\langle L\rangle \neg A$.

4. $\vDash[L] A \supset[L][L] A-$ транзитивность.

5. $\vDash[L][L] A \supset[L] A-$ плотность.

6. $\vDash(\langle L\rangle A \wedge\langle L\rangle B) \supset\langle L\rangle(A \wedge B) \vee\langle L\rangle(A \wedge\langle L\rangle B) \vee\langle L\rangle(\langle L\rangle A \wedge B)$ линейность.

7. $\vDash\langle L\rangle(A \vee \neg A)-$ бесконечность.

8. $\vDash A \Rightarrow \vDash[R] A$. 
9. $\vDash[R](A \supset B) \supset([R] A \supset[R] B)$.

10. $\vDash[R] A \equiv \neg\langle R\rangle \neg A$.

11. $\vDash[R] A \supset[R][R] A-$ транзитивность.

12. $\vDash[R][R] A \supset[R] A-$ плотность.

13. $\vDash(\langle R\rangle A \wedge\langle R\rangle B) \supset\langle R\rangle(A \wedge B) \vee\langle R\rangle(A \wedge\langle R\rangle B) \vee\langle R\rangle(\langle R\rangle A \wedge B)-$ линейность.

14. $\vDash\langle R\rangle(A \vee \neg A)-$ бесконечность.

15. $\vDash\langle L\rangle\langle R\rangle A \equiv\langle R\rangle\langle L\rangle A-$ обратимость.

16. $\not \models\langle L\rangle A \equiv\langle R\rangle A$.

Мы видим, что операторы $\langle L\rangle$ и $\langle R\rangle$ позволяют выразить свойства транзитивности, плотности, линейности и бесконечности, что, в свою очередь, позволяет нам ассоциировать их с временным порядком отдельных процессов. Это находится в полном соответствии с тем, что было сказано в Шалак, 2020. Общезначимость формулы 15 говорит о симметричности левого и правого направлений, что соответствует обратимости времени в законах физических наук. В то же время необщезначимость формулы 16 говорит, что левые части процессов отличны от правых, прошлое отлично от будущего.

\section{2. Состояния и события}

Передо мной на столе лежит открытая книга. Ее состояние - быть открытой. Я могу перелистывать ее страницы, но она продолжает находиться в этом состоянии. Затем я закрываю книгу. Закрывание книги событие, которое характеризуется тем, что наступает оно лишь после того, как книга становится закрытой. Многое в изменяющемся мире можно описать в терминах смены состояний и событий. Состояние - характеристика того, что стабильно в процессе, событие - характеристика происходящих в процессе изменений. Эти понятия не абсолютны. Книга у меня на столе сохраняет состояние быть открытой, но в то же время оно состоит из ряда промежуточных событий перелистывания страниц. Идея различения этих понятий взята из работ по планированию действий Allen, 1984 и Allen, Ferguson, 1994.

Введем в язык еще два оператора, которые обозначим посредством $\langle P\rangle$ и $[P]$. Они позволят делать утверждения не только о началах или концах процессов, но и о любых внутренних фрагментах: 
Def.10 $\langle P\rangle A \Leftrightarrow_{\operatorname{def}}\langle L\rangle A \vee\langle R\rangle\langle L\rangle A \vee\langle R\rangle A$.

Def.11 $[P] A \Leftrightarrow_{\text {def }} \neg\langle P\rangle \neg A$.

Им будут соответствовать условия истинности:

- $x \vDash\langle P\rangle A \Leftrightarrow \exists y(\boldsymbol{P} x y \& y \vDash A)$;

- $x \vDash[P] A \Leftrightarrow \forall y(\boldsymbol{P} x y \Rightarrow y \vDash A)$.

Утверждение 3. Формулы вида $\langle P\rangle A$ и $[P] A$ обладают следующими свойствами:

1. $\vDash A \Rightarrow \vDash[P] A$.

2. $\vDash[P](A \supset B) \supset([P] A \supset[P] B)$.

3. $\vDash[P] A \supset[P][P] A-$ транзитивность.

4. $\vDash[P][P] A \supset[P] A-$ плотность.

5. $\vDash\langle P\rangle(A \vee \neg A)-$ бесконечность.

Теперь мы готовы определить понятия состояния процесса и события. В качестве сокращений введем в язык операторы $S$ (state) и $E$ (event).

Процесс характеризуется состоянием $A$, е.т.е. $A$ истинно как на всем процессе, так и на всех его собственных частях:

$$
\text { Def.12 } S A \Leftrightarrow_{\text {def }} A \wedge[P] A \text {. }
$$

Процесс представляет событие $A$, е.т.е. $A$ истинно на всем процессе, но ложно на всех его собственных частях:

$$
\text { Def.13 } E A \Leftrightarrow_{\text {def }} A \wedge[P] \neg A .
$$

Чтобы проиллюстрировать эти понятия на реальном примере, представим парусное судно, которое плывет против ветра, периодически меняя галсы. Каждая смена галсов является событием. Оно протекает во времени, но наступает лишь после того, как поворот с перебрасыванием парусов на другую сторону завершен. В промежутках между сменами галсов судно находится в состоянии движения в выбранном направлении. Этот процесс описывается последовательностью событий и состояний в том смысле, как мы их определили. 


\section{3. Бинарные модальности}

Онтология процессов и отношение деления $\boldsymbol{D}$ естественным образом приводят к необходимости использования бинарных модальностей, поскольку унарные модальные операторы не позволяют выразить многих интересных логических характеристик процессов. Добавим к языку логики высказываний бинарный оператор $\oplus$ и определим понятие формулы следующим образом:

Def.14 $A:=$ Prop $|\neg A|(A \wedge B) \mid(A \oplus B)$.

В качестве условия истинности для формул вида $A \oplus B$ примем следующее:

$$
\text { - } x \vDash A \oplus B \Leftrightarrow \exists y \exists z(\boldsymbol{D} x y z \& y \vDash A \& z \vDash B) .
$$

Можно провести аналогию между оператором $\oplus$ и оператором временной логики Вригта «And_next» Wright, 1965; Вригт, 1986. У Вригта смысл формулы $\left(A A n d \_n e x t \bar{B}\right)$ заключается в том, что в модели дискретного времени в некоторый момент имеет место $A$, а в следующий момент имеет место $B$. В логике процессов формула $(A \oplus B)$ означает, что при некотором делении процесса на две части на левой имеет место $A$, а на правой $-B$, но это не то же самое, что $B$ имеет место в следующий момент времени. Например, в модели дискретного времени трудно предложить естественную семантику для предложения «Я пришел домой и отдыхаю». В процессуальной логике это выражается непосредственным образом ( $Я$ пришел домой» $\oplus \ll$ « отдыхаю»).

Оператор $\oplus$ обладает чертами некоммутативной конъюнкции и позволяет ввести в язык аналог импликации $\rightarrow$ для обозначения условной связи между предшествующими и последующими частями процессов.

Def.15 $A \rightarrow B \Leftrightarrow \Leftrightarrow_{\text {def }} \neg(A \oplus \neg B)$.

Условия истинности для формул типа $A \rightarrow B$ имеют следующий вид:

$$
\text { - } x \vDash A \rightarrow B \Leftrightarrow \forall y \forall z(\boldsymbol{D} x y z \& y \vDash A \Rightarrow z \vDash B) \text {. }
$$

О выразительных возможностях языка с бинарным оператором $\oplus$ говорит уже то, что в нем можно легко определить рассмотренные ранее унарные операторы:

$$
\begin{aligned}
& \text { Def.16 }\langle L\rangle A \Leftrightarrow_{\text {def }} A \oplus(A \vee \neg A) . \\
& \text { Def.17 }\langle R\rangle A \Leftrightarrow_{\text {def }}(A \vee \neg A) \oplus A .
\end{aligned}
$$


Связка «если..., то...» естественного языка часто употребляется в овремененном смысле для обозначения, например, причинной связи. Обычная материальная импликация для этого не подходит. Оператор $\rightarrow$ обладает характеристиками условной связи, которую можно понимать как причинную связь, когда причина отлична от действия и полностью ему предшествует. Примером такой причинной связи является, например, столкновение биллиардных шаров.

Логика с операторами $\oplus$ и $\rightarrow$ родственна интервальной временной логике $C D T$ Venema, 1991, но в деталях и семантике отличается.

Утверждение 4. Формулы вида $A \oplus B$ и $A \rightarrow B$ обладают следующими свойствами:

1. $\vDash(A \vee \neg A) \oplus(A \vee \neg A)$.

2. $\vDash A \oplus(B \oplus C) \equiv(A \oplus B) \oplus C$.

3. $\not \models A \rightarrow A$.

4. $\vDash A \Rightarrow \vDash B \rightarrow A$.

5. $\vDash A \Rightarrow \vDash \neg A \rightarrow B$.

6. $\vDash A \rightarrow(B \wedge C) \Leftrightarrow \vDash(A \rightarrow B) \wedge(A \rightarrow C)$.

7. $\vDash A \rightarrow B \Rightarrow \vDash(A \wedge C) \rightarrow B$.

8. $\vDash(A \rightarrow C) \wedge(B \rightarrow C) \Rightarrow \vDash(A \vee B) \rightarrow C$.

9. $\vDash A \rightarrow B \Rightarrow \vDash A \rightarrow(B \vee C)$.

10. $\vDash A, \vDash A \rightarrow B \Rightarrow \vDash[R] B$.

11. $\vDash(A \rightarrow B) \wedge\langle L\rangle A \supset\langle R\rangle B$.

12. $\vDash A \rightarrow\langle L\rangle B, \vDash B \rightarrow C \Rightarrow \vDash A \rightarrow\langle R\rangle C$.

Общезначимость формулы 1 соответствует семантическому постулату 1 для отношения $\boldsymbol{D}$ и говорит о том, что любой процесс может быть разделен на две части.

Формула 2 говорит об ассоциативности отношения деления процессов и соответствует постулату 6 для отношения $\boldsymbol{D}$.

Необщезначимость закона тождества 3 следует из постулата 2 для отношения $\boldsymbol{D}$ и независимости приписывания истинностных значений пропозициональным переменным на частях процессов. 
Формулы 4 и 5 являются аналогами парадоксов материальной импликации, но не для истинности, а для общезначимости формулы $A$.

Формулы 6-9 не нуждаются в комментариях.

Формулы 10 представляют аналог modus ponens для общезначимости: если общезначимы $A$ и $A \rightarrow B$, то в конце каждого процесса будет истинHо $B$.

Формула 11 является сильным вариантом 10, более детально представляя внутреннюю структуру modus ponens. Чтобы применить его, формула $A$ должна быть истинна в начале текущего процесса, и тогда в конце процесса будет истинна формула $B$.

Формулы 12 представляют транзитивность условной связи $\rightarrow$ на процессах.

\section{3. Динамика процессов}

В предыдущих параграфах мы рассмотрели формулы с модальными операторами, истинность которых зависит лишь от истинности на частях данного процесса, но не зависит от возможного последующего его развития. Это развитие может быть обусловлено либо причинными связями, которые естественным образом приводят к продолжению текущих процессов, либо действиями активных агентов, которые сами выбирают, как дальше будет развиваться данный процесс.

\section{1. Продолжение процессов}

Добавим к языку с бинарными модальными операторами $\oplus$ и $\rightarrow$ новый унарный оператор $\diamond$ :

Def.18 $A:=$ Prop $|\neg A|(A \wedge B)|(A \oplus B)|(A \rightarrow B) \mid \diamond A$.

Следующим образом определим условия истинности формул вида $\diamond A$ :

$$
\text { - } x \vDash \diamond A \Leftrightarrow \exists p \exists y(D p x y \& y \vDash A) \text {. }
$$

Смысл формул вида $\diamond A$ очевиден. $\diamond A$ истинна на текущем процессе, е.т.е. этот процесс имеет продолжение, на котором истинна формула $A$. Это определение замечательно тем, что оно задает направленность развития процессов, и потому допустимо читать формулы вида $\diamond A$ как «далее будет $A$ ». Итерацию оператора $\diamond$ можно понимать просто как будет.

Добавим к языку новый оператор $\square$ в качестве дуала для $\diamond$ :

$$
\begin{aligned}
& \text { Def.19 } \square A \Leftrightarrow \text { def } \neg \diamond \neg A . \\
& \text { • } x \vDash \square A \Leftrightarrow \forall p \forall y(D p x y \Rightarrow y \vDash A) .
\end{aligned}
$$


Утверждение 5. Имеют место следующие утверждения:

1. $\vDash A \Rightarrow \vDash \square A$.

2. $\vDash \square(A \supset B) \supset(\square A \supset \square B)$.

3. $\not \models \square A \supset \diamond A$.

4. $\vDash A \rightarrow B \Rightarrow \vDash A \supset \square B$.

Истинность формулы 4 говорит о том, что если общезначима условная связь $A \rightarrow B$ и на каком-то процессе истинно $A$, то на каждом его продолжении будет истинно $B$. Однако необщезначимость формулы 3 говорит, что из $\square B$ еще не следует, что процесс действительно будет иметь продолжение. Образно говоря, онтология процессов допускает их окончание.

\section{2. Порождение процессов}

В предыдущем параграфе мы рассмотрели ситуацию продолжения процессов в общем виде независимо от того, чем это продолжение вызвано. Теперь же рассмотрим случай, когда продолжение текущего процесса имеет место в силу действий активного агента. Пусть он фиксирован и с ним ассоциирован набор элементарных действий Act, которые он способен совершить.

Распространим функцию интерпретации $\boldsymbol{I}$ на элементарные действия $\boldsymbol{I}$ : $A c t \cup$ Prop $\rightarrow 2^{\mathbf{U}}$. Каждому действию $d \in A c t$ эта функция сопоставляет множество процессов $\boldsymbol{I}(d)$, которые оно может породить.

Понятие формулы определим следующим образом:

Def.20 $A:=$ Prop $|\neg A|(A \wedge B)|(A \oplus B)|(A \rightarrow B)|\diamond A|<d>A$, где $d \in$ Act.

В качестве условий истинности для формул вида $<d>A$ примем следующие:

- $x \vDash<d>A \Leftrightarrow \exists p \exists y(\boldsymbol{D} p x y \& y \in \boldsymbol{I}(d) \& y \vDash A)$.

Определим дуальный оператор $[d]$ и приведем для него соответствующие условия истинности:

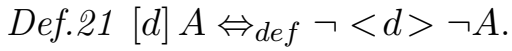

- $x \vDash[d] A \Leftrightarrow \forall p \forall y(\boldsymbol{D} p x y \& y \in \boldsymbol{I}(d) \Rightarrow y \vDash A)$. 
Утверждение 6. Имеют место следующие утверждения:

1. $\vDash A \Rightarrow \vDash[d] A$.

2. $\vDash[d](A \supset B) \supset([d] A \supset[d] B)$.

3. $\vDash[d] A \supset \diamond A$.

Общезначимость формулы 3 говорит о том, что порождение новых процессов в результате действий активных агентов является частным случаем общего продолжения процессов.

\section{3. Целеполагание активных агентов}

Если посмотреть на происходящие в окружающем нас мире изменения, их можно разделить на два вида. K первому виду относятся изменения, описываемые законами наук. Они были эксплицированы в номологической модели объяснения К. Гемпеля. Ко второму виду относятся изменения, инициируемые активными целесообразно действующими агентами. Такие изменения могут быть названы алгоритмическими, поскольку получают объяснение в терминах следования предписаниям для достижения требуемого результата. Примером первого вида изменений может быть падение камня с крыши, второго - бросок камня, чтобы попасть в цель. В первом случае падение камня описывается законами механики, во втором целесообразным действием человека.

Широко распространено мнение, что неформальное понятие алгоритма было строго уточнено в работах А. Чёрча и А. Тьюринга, что тезис ЧёрчаТьюринга является утверждением о том, что любой алгоритм может быть представлен в терминах абстрактного вычислительного устройства Тьюринга. Это не совсем верно. Тьюринг предложил математический формализм для описания возможных символьных преобразований, которые способен произвести человек-вычислитель, но не описание общего понятия алгоритма. Человек-вычислитель оперирует с символами, а не с физическими объектами. Знаконосители всего лишь репрезентируют то, что человек хочет видеть за ними, а вычисление на машине Тьюринга - это переход от одной символьной репрезентации, называемой начальной, к другой, называемой конечной. В зависимости от принятых соглашений одна и та же комбинация букв используемого алфавита может означать разные объекты. Например, три единички 111 по их количеству могут представлять число три, в двоичной системе записи они будут представлять уже число семь, а в десятичной - число сто одиннадцать. Соответственно, в зависимости от принятых соглашений одна и та же машина Тьюринга будет вычислять разные функции. 
С другой стороны, машина Тьюринга описывается в языке с конечным алфавитом символов, внутренних состояний и действий. С их помощью формулируются конечные наборы правил, управляющих работой машины. Это позволяет путем гёделизации закодировать все возможные машины и дальше исследовать их характеристики в терминах Гёделевых номеров, которые однозначно представляют не только синтаксис, но и семантику машины Тьюринга. Результат работы машины Тьюринга однозначно детерминирован ее номером (программой) и входными данными. Именно это и используется для доказательства основных теорем теории вычислимости.

Всякое действие, выполняемое абстрактной машиной Тьюринга, завершается успешно. У нее не могут закончиться чернила для печати, и никто не может вдруг выключить ее или сломать. Результат любого действия совпадает с целью, ради которой оно совершается. Если машина должна напечатать символ на рабочей ленте, то она его печатает, и результат выполнения всей программы будет одним и тем же при каждом ее запуске.

Теперь посмотрим на алгоритмические явления в природе, которые внешним образом мы можем описать как следование набору предписаний для достижения требуемого результата. Любой такой набор предписаний можно переформулировать в виде набора правил «Если $\boldsymbol{C}$, сделай $\boldsymbol{d}$ ». Легко показать, как с помощью таких правил задается очередность выполнения действий, их повторение, выбор вариантов, начало и завершение алгоритма. Именно с помощью таких условных правил формулируются программы для машины Тьюринга. Если мы захотим описать физически реализуемые алгоритмы таким же образом, то обнаружим, что в правилах не хватает указания на цели, ради которых они совершаются, и потому говорить об успешности выполнения правила нельзя без учета достижения цели. Более правильным было бы представлять правила, описывающие

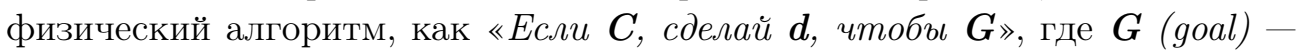
некоторое событие или процесс, являющиеся целью выполнения данного правила. Например, «Если ты голоден $(\boldsymbol{C})$, пойди в магазин (d), чтобъ купить продукты $(\boldsymbol{G}) »$. Покупка продуктов - это физический результат, а не символьная репрезентация в виде кассового чека. В этом заключается главное отличие символьных алгоритмов от физических. «Если в комнате темно $(\boldsymbol{C})$, нажми на кнопку выключателя (d), чтобы зажечь лампу $(G) »$. Горящая лампа - это конкретный физический процесс, а не символьная репрезентация. Если болит голова, мы выпиваем таблетку обезболивающего, чтобы запустить специальные физико-химические процессы в организме. Крестьяне пашут землю и бросают в нее зерна, чтобы запустить процесс созревания урожая. 
Алгоритмические процессы в природе выполняются в окружении неалгоритмических процессов. Неожиданный порыв ветра может помешать попасть камнем в цель, хлеба в магазине может не оказаться, электропроводка в квартире может оказаться повреждена, капризы погоды могут загубить весь урожай. Выполнение одних и тех же действий вчера и сегодня не гарантирует получения того же результата. В этом заключается еще одно отличие природных алгоритмов от символьных. Они всегда выполняются в новом физическом контексте, а теория хаоса учит, что иногда даже малейшие изменения в условиях могут приводить к непредсказуемым последствиям. Поэтому, выполнив действие $\boldsymbol{d}$, мы не можем быть уверены, что наступит $\boldsymbol{G}$.

Чтобы иметь возможность описывать целесообразные действия агентов, возьмем язык с трехместным оператором <;;> и определим понятие формулы следующим образом:

Def.22 $A:=$ Prop $|\neg A|(A \wedge B)|(A \oplus B)|(A \rightarrow B)|\diamond A|<d>A \mid$ $<C ; d ; G>A$.

В качестве условия истинности примем, что на процессе х после выполнения действия $\langle C ; d ; G>$ истинна формула $A$, е. и т.е. на процессе $x$ истинны условия $C$ применения данного правила, в результате действия $d$ существует продолжение $y$ процесса $x$, и на этом продолжении $y$ истинно $A$ :

- $x \vDash<C ; d ; G>A \Leftrightarrow \exists p \exists y(\boldsymbol{D} p x y \& x \vDash C \& y \in \boldsymbol{I}(d) \& y \vDash A)$.

Обычным образом мы можем ввести в язык и дуал:

Def.23 $[C ; d ; G] A \Leftrightarrow_{\text {def }} \neg<C ; d ; G>\neg A$.

- $x \vDash[C ; d ; G] A \Leftrightarrow \forall p \forall y(\boldsymbol{D} p x y \& x \vDash C \& y \in \boldsymbol{I}(d) \Rightarrow y \vDash A)$.

Первое, что бросается в глаза, - в условиях истинности формул вида $<C ; d ; G>A$ не фигурирует цель $G$ выполнения этого правила. Но этого в общем случае и не должно быть. Выполнение любого правила всегда приводит к какому-то результату, и это отражено в определении, а достижение или недостижение цели - это оценка успешности или неуспешности его выполнения. Чтобы говорить об этом в языке, достаточно ввести следующее сокращение:

$$
\text { Def.24 } !<C ; d ; G>A \Leftrightarrow_{d e f}<C ; d ; G>(A \& G) .
$$

Подробный анализ построенной логики планируется сделать в последующих публикациях, посвященных теме построения теории физических (природных) алгоритмических процессов. 


\section{4. Заключение}

Классическая логика высказываний, которую можно рассматривать как логику субстанциальной онтологии, проста и единственна с точностью до дефинициальной эквивалентности других формулировок. Исходные объекты процессуальной онтологии обладают внутренней структурой, связанной с их протяженностью и направленностью. Чтобы иметь возможность рассуждать о процессах, требуются языки с более богатыми выразительными возможностями по сравнению с языками классической логики. Эти языки неявно предполагают понятие времени, как производного от протяженности и направленности процессов.

Очевидное деление процессов на два вида - каузально-динамические и агентно-зависимые - требует учета существования активных действующих агентов, что приводит к необходимости построения вариантов логик действия. Важной темой в развитии логики процессуальной онтологии является тема построения теории физических (природных) алгоритмических процессов. В настоящее время она в основном разрабатывается как вспомогательная в рамках работ по построению роботизированных устройств и планированию действий в ИИ. Привлечение к данной теме внимания логиков-философов может позволить взглянуть на нее с более общей точки зрения.

\section{Литература}

Вригт, 1986 - Вригт Г.X. фон. Время, изменение и противоречие. Логикофилософские исследования. Избр. тр. М.: Прогресс, 1986. С. 513-538.

Шалак, 2020 - Шалак В.И. Сравнительный анализ процессуальной и субстанциальной онтологий // Логические исследования / Logical Investigations. 2020. T. 26. № 2. C. 58-86. DOI: 10.21146/2074-1472-2020-26-2-58-86.

Allen, 1984 - Allen J.F. Towards a General Theory of Action and Time // Artificial Intelligence. 1984. Vol. 23. P. 123-154.

Allen, Ferguson, 1994 - Allen J.F., Ferguson G. Actions and Events in Interval Temporal Logic // Journal of Logic and Computation. 1994. Vol. 4. Is. 5. P. 531579. DOI: $10.1093 / \log c o m / 4.5 .531$.

Venema, 1991 - Venema Y. A Modal Logic for Chopping Intervals // Journal of Logic and Computation. 1991. Vol. 1. Is. 4. P. 453-476. DOI: 10.1093/logcom/1.4.453.

Wright, 1965 - Wright G.H. von. And next // Acta philosophica Fennica. 1965. № 18. P. 293-304. 


\title{
VLADimir I. SHALACK \\ Logic in the process ontology
}

\author{
Vladimir I. Shalack \\ Institute of Philosophy of Russian Academy of Sciences, \\ 12/1 Goncharnaya St., Moscow, 109240, Russian Federation. \\ E-mail: shalack@gmail.com
}

\begin{abstract}
The aim of the work is to study the language tools that can be used to conduct arguments about the processual ontology, which differs from the substantial more complex structure of the elementary "bricks" that underlie it. As a result, it becomes necessary to use languages that have richer expressive capabilities than the language of classical logic. In this paper, we consider languages with unary and binary modalities. Individual processes can be analyzed from two points of view-statics and dynamics. In the first case, we are interested in statements whose truth depends only on the internal structure of the current process. The relation of division of the processes allows us to determine on its parts a transitive dense linear order without the first and last elements, which can be understood as the local time order of this process. This allows us to talk about the beginning of processes, their flow and end. It becomes possible to define the concepts of process state and process as events. The inclusion of a binary modality such as a noncommutative conjunction in the language allows us to define an analog of a causal-like conditional relationship between parts of the process. The dynamics of processes implies the possibility of their continuation beyond the current process. This can happen either due to implicit causal relationships, when the current process contains the cause for its future continuation, or as a result of the actions of active agents who themselves construct this continuation. To represent the dynamics of processes as a consequence of the actions of active agents, we use a simplified version of the dynamic logic language. Also it's shown how you can take into account the goals that active agents set for themselves when performing certain actions.
\end{abstract}

Keywords: process, process ontology, process logic, process statics, process dynamics

For citation: Shalack V.I. "Logika v ontologii protsessov" [Logic in the process ontology], Logicheskie Issledovaniya / Logical Investigations, 2021, Vol. 27, No. 2, pp. 48-65. DOI: 10.21146/2074-1472-2021-27-2-48-65 (In Russian)

\section{References}

Allen, 1984 - Allen, J.F. "Towards a General Theory of Action and Time", Artificial Intelligence, 1984, Vol. 23, pp. 123-154.

Allen, Ferguson, 1994 - Allen, J.F., Ferguson, G. "Actions and Events in Interval Temporal Logic", Journal of Logic and Computation, 1994, Vol. 4, Is. 5, pp. 531579. DOI: $10.1093 / \log c o m / 4.5 .531$. 
Shalack, 2020 - Shalack, V.I. "Sravnitel'nyi logicheskii analiz substantsial'noi i protsessual'noi ontologii" [Comparative logical analysis of substantive and processual ontologies], Logical Investigations, 2020, Vol. 26, No. 2, pp. 58-86. DOI: 10.21146/2074-1472-2020-26-2-58-86. (In Russian)

Venema, 1991 - Venema, Y. "A Modal Logic for Chopping Intervals", Journal of Logic and Computation, 1991, Vol. 1, Is. 4, pp. 453-476. DOI: 10.1093/logcom/1.4.453.

Wright, 1965 - von Wright, G.H. "And next", Acta philosophica Fennica, 1965, No. 18, pp. 293-304.

Wright, 1986 - von Wright, G.H. "Vremya, izmenenie i protivorechie" [Time, change, and contradiction], in Logiko-filosofskie issledovaniya [Philosophical papers]. Moscow: Progress, 1986, pp. 513-538. (In Russian) 